\title{
Renormalization of the Inverse Square Potential
}

\author{
Horacio E. Camblong, ${ }^{1}$ Luis N. Epele, ${ }^{2}$ Huner Fanchiotti, ${ }^{2}$ and Carlos A. García Canal ${ }^{2}$ \\ ${ }^{1}$ Department of Physics, University of San Francisco, San Francisco, California 94117-1080 \\ ${ }^{2}$ Laboratorio de Física Teórica, Departamento de Física, Universidad Nacional de La Plata, C.C. 67 - 1900 La Plata, Argentina
}

(Received 2 March 2000)

\begin{abstract}
The quantum-mechanical $D$-dimensional inverse square potential is analyzed using field-theoretic renormalization techniques. A solution is presented for both the bound-state and scattering sectors of the theory using cutoff and dimensional regularization. In the renormalized version of the theory, there is a strong-coupling regime where quantum-mechanical breaking of scale symmetry takes place through dimensional transmutation, with the creation of a single bound state and of an energy-dependent $s$-wave scattering matrix element.
\end{abstract}

PACS numbers: 03.65.Ge, 03.65.Nk, 11.10.Gh, 31.15.-p

The quantum-mechanical inverse square potential is a singular problem that has generated controversy for decades. For instance, the solution proposed in Ref. [1] failed to give a Hamiltonian bounded from below, and this led to a number of alternative regularization techniques [2-4] based on appropriate parametrizations of the potential-including the replacement [5] of selfadjointness by an interpretation of the "fall of the particle to the center" [6]. However, it is generally recognized that the singular nature of this problem lies in that its Hamiltonian, being symmetric but not self-adjoint, admits self-adjoint extensions [7]. Recently, a renormalized solution was presented using field-theoretic techniques [8], but it was just limited to the one-dimensional case and cutoff renormalization.

In this Letter (i) we generalize the results of Ref. [8] to $D$ dimensions (including the all-important $D=3$ case) using cutoff regularization in configuration space; (ii) present a complete picture of the renormalized theory; and (iii) confirm the same conclusions using dimensional regularization [9]. This problem is crucial for the analysis and interpretation of the point dipole interaction of molecular physics [10,11], and may be relevant in polymer physics [12]. In addition (i) it displays remarkable similarities with the two-dimensional $\delta$-function potential [13-15]; (ii) it provides another example of dimensional transmutation [16] in a system with a finite number of degrees of freedom; and (iii) it illustrates the relevance of field-theoretic concepts in quantum mechanics [13-15,17].

This problem is ideally suited for implementation in configuration space [18], where the radial Schrödinger equation for a particle subject to the $r^{-2}$ potential in $D$ dimensions [19] reads (with $\hbar=1$ and $2 m=1$ )

$$
\begin{aligned}
& {\left[\frac{1}{r^{D-1}} \frac{d}{d r}\left(r^{D-1} \frac{d}{d r}\right)+E\right.} \\
& \left.\quad-\frac{l(l+D-2)-\lambda}{r^{2}}\right] R_{l}(r)=0,
\end{aligned}
$$

which is explicitly scale invariant because $\lambda$ is dimensionless [20]. In Eq. (1), $l$ is the angular momentum quantum number and $\lambda>0$ corresponds to an attractive potential; with the transformation $R_{l}(r)=r^{-(D-1) / 2} u_{l}(r)$, Eq. (1) is recognized to have solutions $R_{l}(r)=r^{-(D / 2-1)} Z_{s_{l}}(\sqrt{E} r)$, where $Z_{s_{l}}(z)$ represents an appropriate linear combination of Bessel functions of order $s_{l}=\left[\lambda_{l}^{(*)}-\lambda\right]^{1 / 2}$, with

$$
\lambda_{l}^{(*)}=(l+D / 2-1)^{2} .
$$

If $\lambda$ were allowed to vary, one would see that the nature of the solutions changes around the critical value $\lambda_{l}^{(*)}$, for each angular momentum state. For $\lambda<\lambda_{l}^{(*)}$ (including repulsive potentials), the order $s_{l}$ of the Bessel functions is real, so that the solution regular at the origin is proportional to the Bessel function of the first kind $J_{S_{l}}(\sqrt{E} r)$. However, the same solution fails to satisfy the required behavior at infinity for bound states $(E<0)$; in other words, in the weak-coupling regime, the potential cannot sustain bound states. Moreover, the scattering solutions are scale invariant [20], with $D$-dimensional phase shifts $\delta_{l}^{(D)}=\left\{\left[\lambda_{l}^{(*)}\right]^{1 / 2}-\left[\lambda_{l}^{(*)}-\lambda\right]^{1 / 2}\right\} \pi / 2$. Nothing is surprising here: the potential $r^{-2}$ is explicitly scale invariant and no additional scale arises at the level of the solutions, which are well-behaved-one could say that the potential looks like a regular "repulsive" one. However, this picture changes dramatically for $\lambda>\lambda_{l}^{(*)}$ : all the Bessel functions acquire an uncontrollable oscillatory character through the imaginary order $s_{l}=i \Theta_{l}$, where $\Theta_{l}=\left[\lambda-\lambda_{l}^{(*)}\right]^{1 / 2}$, as we shall see next.

For the remainder of this Letter, we will mainly analyze the strong-coupling regime $\lambda>\lambda_{l}^{(*)}$. First, for the boundstate sector, from Eq. (1), $u_{l}(r) \propto \sqrt{r} K_{i \Theta_{l}}(\sqrt{|E|} r)$, with $K_{s_{l}}(z)$ being the modified Bessel function of the second kind [21], whose behavior near the origin is of the form

$$
\begin{aligned}
K_{i \Theta_{l}}(z) \stackrel{(z \rightarrow 0)}{\sim} & -\sqrt{\frac{\pi}{\Theta_{l} \sinh \left(\pi \Theta_{l}\right)}} \sin \left[\Theta_{l} \ln \left(\frac{z}{2}\right)-\delta_{\Theta_{l}}\right] \\
& \times\left[1+O\left(z^{2}\right)\right]
\end{aligned}
$$

where $\delta_{\Theta_{l}}$ is the phase of $\Gamma\left(1+i \Theta_{l}\right)$. In Eq. (3), the wave function oscillates with a monotonically increasing 
frequency as $r \rightarrow 0$. As a result, there is no criterion for the selection of a particular subset of states and the boundstate spectrum is continuous and not bounded from below. Clearly, the problem should be renormalized in such a way that the Hamiltonian recovers its self-adjoint character [7].

A first attempt [1,22] is to use Eq. (3) and recognize that the orthogonality condition for the eigenstates restores the discrete nature of the spectrum; unfortunately, in this approach, the Hamiltonian is not bounded from below. However, as was proposed in Ref. [8] for the particular simple case $D=1$, Eq. (3) can be regularized by introducing a short-distance cutoff $a$, with $a \ll|E|^{-1 / 2}$, so that the regular boundary condition $u_{l}(a)=0$ is implemented in lieu of the undefined behavior at $r=0$. Then, Eq. (3) gives the zeros of the modified Bessel function of the second kind with imaginary order, $z_{n}=2 e^{\left(\delta_{\Theta_{l}}-n \pi\right) / \Theta_{l}}$ [up to a correction factor $1+O\left(z_{n}^{2} / \Theta_{l}\right)$ ], where $n$ is an integer; moreover, the assumption that $z_{n} \ll 1$, with $\Theta_{l} \geq 0$, implies that $(-n)<0$, with the conclusion that $n=1,2,3, \ldots$. Parenthetically, $z_{n} \ll 1$ only if $\Theta_{l} \ll 1$, so that $\delta_{\Theta_{l}}=-\gamma \Theta_{l}+O\left(\Theta_{l}^{2}\right)$ (with $\gamma$ being the EulerMascheroni constant) and the energy levels become

$$
E_{n_{r} l}=-\left(\frac{2 e^{-\gamma}}{a}\right)^{2} \exp \left(-\frac{2 \pi n_{r}}{\Theta_{l}}\right),
$$

where $n=n_{r}$ stands for the radial quantum number.

Equation (4) should now be renormalized by requiring that $\Theta_{l}=\Theta_{l}(a)$ in the limit $a \rightarrow 0$. More precisely, in order for the ground state [characterized by the quantum numbers $\left.(\mathrm{gs}) \equiv\left(n_{r}=1, l=0\right)\right]$ to "survive" the renormalization prescription with a finite energy, it is required that $\Theta_{(\mathrm{gs})}(a) \stackrel{(a \rightarrow 0)}{\longrightarrow} 0^{+}$. This condition amounts to a "critically strong" coupling, $\lambda(a) \stackrel{(a \rightarrow 0)}{\longrightarrow} \lambda_{(\mathrm{gs})}^{(*)}+0^{+}$(where the notation $\Theta_{0}=\Theta_{(\mathrm{gs})}$ and $\lambda_{0}^{(*)}=\lambda_{(\mathrm{gs})}^{(*)}$ is understood for the ground state). In particular, with this ground-state renormalization, the required relation between $\Theta_{(\mathrm{gs})}(a)$ and $a$, for $a$ small, is

$$
-g^{(0)}=\frac{2 \pi}{\Theta_{(\mathrm{gs})}(a)}+2 \ln \left(\frac{\mu a}{2}\right)+2 \gamma,
$$

where $\mu$ is an arbitrary renormalization scale with dimensions of inverse length and $g^{(0)}$ is an arbitrary finite part associated with the coupling, such that

$$
E_{(\mathrm{gs})}=-\mu^{2} \exp \left[g^{(0)}\right] \leadsto-\mu^{2} .
$$

In Eq. (6), it is understood that, due to the arbitrariness of both $g^{(0)}$ and $\mu$, the simple choice $g^{(0)}=0$ can be made. Finally, the ground-state wave function is obtained in the limit $\Theta_{(\mathrm{gs})}(a) \stackrel{(a \rightarrow 0)}{\longrightarrow} 0^{+}$, which yields [23]

$$
\Psi_{(\mathrm{gs})}(\mathbf{r})=\sqrt{\Gamma\left(\frac{D}{2}\right)\left(\frac{\mu^{2}}{\pi}\right)^{D / 2}} \frac{K_{0}(\mu r)}{(\mu r)^{D / 2-1}},
$$

whose functional form, up to a factor $r^{-(D / 2-1)}$, is dimensionally invariant [24].

The existence of a ground state with a dimensional scale $\mu \propto\left|E_{(\mathrm{gs})}\right|^{1 / 2}$ violates the manifest scale invariance of the theory defined by Eq. (1), but its magnitude is totally arbitrary and spontaneously generated by renormalization. Here we recognize the fingerprints of dimensional transmutation [16].

The next question refers to the possible existence of excited states in the renormalized theory. For any hypothetical state with angular momentum quantum number $l>0$, this question can be straightforwardly answered from the ground-state renormalization condition $\Theta_{(\mathrm{gs})}(a) \stackrel{(a \rightarrow 0)}{\longrightarrow} 0^{+}$, which, together with Eq. (2), provides the inequality $\lambda=$ $\lambda_{(\mathrm{gs})}^{(*)}=(D / 2-1)^{2}<\lambda_{l}^{(*)}$. Then, if such a state existed, it would automatically be pushed into the weak-coupling regime, with the implication that it could not survive the renormalization process. This means that there are no excited states with $l>0$. Next, the question arises as to the possible existence of bound states with $l=0$ and $n_{r} \neq 0$. The fact that these hypothetical bound states also cease to exist in the renormalized theory follows from the exponential suppression

$$
\left|\frac{E_{n_{r} 0}}{E_{(\mathrm{gs})}}\right|=\exp \left[-\frac{2 \pi\left(n_{r}-1\right)}{\Theta_{(\mathrm{gs})}}\right] \stackrel{\left(\Theta_{(\mathrm{gs})} \rightarrow 0, n_{r}>1\right)}{\longrightarrow} 0 .
$$

Moreover, it is easy to see that, for these hypothetical states, the corresponding limit of the wave function becomes ill defined, so that they effectively vanish. In conclusion, the renormalization process annihilates all candidates for a renormalized bound state, with the only exception of the ground state of the regularized theory, which acquires the finite energy value (6) and the normalized wave function (7).

Similarly, the scattering solutions can be studied by going back to Eq. (1), which implies that $u_{l}(r) / \sqrt{r}$ is a linear combination of the Hankel functions $H_{i \Theta_{l}}^{(1,2)}(k r)$ [21], whose asymptotic behavior $(r \rightarrow \infty)$, combined with the regularized boundary condition $u_{l}(a)=0$, provides the scattering matrix elements $S_{l}^{(D)}(k ; a)$ and phase shifts $\delta_{l}^{(D)}(k ; a)$. For example, the phase function $\phi_{l}^{(D)}(k ; a)=$ $\delta_{l}^{(D)}(k ; a)-(l+D / 2-1) \pi / 2$ is given by

$$
\tan \left(\phi_{l}^{(D)}(k ; a)\right)=\tanh \left(\frac{\pi \Theta_{l}}{2}\right) \frac{1-\mathcal{T}_{l}(k ; a) \varrho_{l}}{\mathcal{T}_{l}(k ; a)+\varrho_{l}},
$$

where $\mathcal{T}_{l}(k ; a)=\tan \left[\Theta_{l} \ln (k a / 2)\right]$ and $\varrho_{l}=v_{-, l} / i v_{+, l}$, with $v_{ \pm, l}=\Gamma\left(1-i \Theta_{l}\right) \pm \Gamma\left(1+i \Theta_{l}\right)$. Equation (9) is ill defined in the limit $a \rightarrow 0$; in effect, the variable $\mathcal{T}_{l}(k ; a)$ oscillates wildly between $-\infty$ and $\infty$, unless $\Theta_{l} \rightarrow 0$, just as for the bound-state sector. From Eqs. (5) and (6), for $l=0$, in the limit $a \rightarrow 0$, the renormalized $s$-wave phase shift becomes 


$$
\tan \left(\delta_{0}^{\left(D_{0}\right)}(k)-(D / 2-1) \pi / 2\right)=\frac{\pi}{\ln \left(k^{2} /\left|E_{(\mathrm{gs})}\right|\right)}
$$

Equation (10) explicitly displays the scattering behavior of $s$ states, as well as its relation with the bound-state sector of the theory. Both the functional form of Eq. (10) and the existence of a unique bound state in the renormalized theory are properties shared by the two-dimensional $\delta$ function potential [13-15].

The analysis leading to Eq. (10) refers to $l=0$. For all other angular momenta, $l>0$, the coupling will be weak, so that the phase shifts will be given by their unregularized values, with the condition that $\lambda=\lambda_{(\mathrm{gs})}^{(*)}=(D / 2-1)^{2}$; then,

$$
\left.\delta_{l}^{(D)}\right|_{l \neq 0}=[(l+D / 2-1)-\sqrt{l(l+D-2)}] \frac{\pi}{2},
$$

which is a scale-invariant expression.

We now turn to an outline of a similar analysis using dimensional renormalization [9]. In particular, we will focus on the bound-state sector of the theory, to illustrate and emphasize the fact that proper renormalization using different regularizations yields the same physics. In this alternative regularization scheme, we define the dimensionally regularized potential in $D^{\prime}$ dimensions in terms of its momentum-space expression, according to [23]

$$
\begin{aligned}
V^{\left(D^{\prime}\right)}\left(r^{\prime}\right) & =-\lambda_{B} \int \frac{d^{D^{\prime}} k^{\prime}}{(2 \pi)^{D^{\prime}}} e^{i \mathbf{k}^{\prime} \cdot \mathbf{r}^{\prime}}\left[\int d^{D} r e^{-i \mathbf{k} \cdot \mathbf{r}} \frac{1}{r^{2}}\right]_{\mathbf{k}=\mathbf{k}^{\prime}} \\
& =-\lambda_{B} \pi^{\epsilon / 2} \Gamma(1-\epsilon / 2) /\left(r^{\prime}\right)^{2-\epsilon},
\end{aligned}
$$

where $\epsilon=D-D^{\prime}$ and $\lambda_{B}$ is the dimensional bare coupling, which will be rewritten as $\mu_{B}=\lambda \mu^{\epsilon}$, with $[\lambda]=1$ and $\mu$ being the floating renormalization scale. The corresponding $D^{\prime}$-dimensional Schrödinger equation for the reduced radial wave function $u_{l}(r)=r^{\left(D^{\prime}-1\right) / 2} R_{l}(r)$ can be converted, by means of a duality transformation $[4,25]$

$$
\left\{\begin{array}{l}
|E|^{1 / 2} r=z^{2 / \epsilon}, \\
|E|^{-D^{\prime} / 4} u_{l}(r)=w_{l, \epsilon}(z) z^{1 / \epsilon-1 / 2},
\end{array}\right.
$$

into

$$
\left\{\frac{d^{2}}{d z^{2}}+\tilde{\eta}-\widetilde{\mathcal{V}}_{\epsilon}(z)-\frac{p^{2}-1 / 4}{z^{2}}\right\} w_{l, \epsilon}(z)=0,
$$

where $\widetilde{\mathcal{V}}_{\epsilon}(z)=-4 \operatorname{sgn}(E) z^{4 / \epsilon-2} / \epsilon^{2}$. In Eq. (14), the new parameters are

$$
\tilde{\eta}=\frac{4 \lambda \pi^{\epsilon / 2} \Gamma(1-\epsilon / 2)}{\epsilon^{2}}\left(\frac{|E|}{\mu^{2}}\right)^{-\epsilon / 2},
$$

and $p=2\left(l+D^{\prime} / 2-1\right) / \epsilon$. The key to solving Eq. (14) is that (i) the parameter $p$ is asymptotically infinite; and (ii) the term $\widetilde{\mathcal{V}}_{\epsilon}(z)$ in Eq. (14) behaves as an infinite hyperspherical potential well in the limit $\epsilon \rightarrow 0$. Then, for bound states, as a first approximation, the particle is trapped in a well with a smooth left boundary proportional to $1 / z^{2}$ and an infinite-well boundary at $z_{2} \approx 1$; as the left turning point is $z_{1} \approx p / \tilde{\eta}^{1 / 2}$, the WKB quantization condition-which we expect to be asymptotically correct for $p \rightarrow \infty$-becomes

$$
\int_{p / \tilde{\eta}^{1 / 2}}^{1} \sqrt{\tilde{\eta}-\frac{p^{2}-1 / 4}{z^{2}}} d z \approx\left(n_{r}-\frac{1}{4}\right) \pi,
$$

so that $\tilde{\eta}^{1 / 2}=p+C_{n_{r}} p^{1 / 3}$, where $C_{n_{r}}=\left[3 \pi\left(n_{r}-\right.\right.$ $1 / 4)]^{2 / 3} / 2$. Therefore, from Eq. (15), it follows that the regularized energies are

$$
\left|E_{n_{r} l}\right|=\mu^{2}\left[\frac{\lambda}{\lambda_{l}^{(*)}}\right]^{2 / \epsilon} \exp \left[G_{n_{r} l}(\epsilon)\right],
$$

where

$$
\begin{aligned}
G_{n_{r} l}(\epsilon)= & -2^{4 / 3} C_{n_{r}}\left(\lambda_{l}^{(*)}\right)^{-1 / 3} \epsilon^{-1 / 3} \\
& +\left[\ln \pi+\gamma+2\left(\lambda_{l}^{(*)}\right)^{-1 / 2}\right] .
\end{aligned}
$$

Equation (17) can be renormalized by demanding that it be finite for the ground state and by letting $\lambda=\lambda(\epsilon)$; explicitly,

$$
\lambda(\epsilon)=\lambda_{(\mathrm{gs})}^{(*)}\left\{1+\frac{\epsilon}{2}\left[g^{(0)}-G_{(\mathrm{gs})}(\epsilon)\right]\right\}+o(\epsilon),
$$

with an arbitrary finite part $g^{(0)}$. In particular, $\lambda(\epsilon) \stackrel{(\epsilon \rightarrow 0)}{\longrightarrow} \lambda_{(\mathrm{gs})}^{(*)}+0^{+}$, i.e., upon renormalization, the coupling becomes critically strong with respect to $s$ states. Just as for cutoff regularization, it follows that only bound states with $l=0$ survive the renormalization process. As for the excited states with $l=0$ in Eq. (17), they are exponentially suppressed according to

$$
\begin{aligned}
\left|\frac{E_{n_{r} 0}}{E_{(\mathrm{gs})}}\right|= & \exp \left[-2^{4 / 3}\left(C_{n_{r}}-C_{1}\right)\left(\lambda_{(\mathrm{gs})}^{(*)}\right)^{-1 / 3} \epsilon^{-1 / 3}\right] \\
& \stackrel{\left(\epsilon \rightarrow 0, n_{r}>1\right)}{\longrightarrow} 0 .
\end{aligned}
$$

Parenthetically, the regularized energies of Eqs. (4) and (17), for finite $a$ and $\epsilon$, are noticeably different; nonetheless, as expected, their renormalized counterparts have exactly the same informational content.

In short, we have analyzed the inverse square potential and found that (i) a critical coupling divides the possible behaviors into two regimes; (ii) in the strong-coupling regime, the theory is ill defined and requires renormalization; and (iii) upon renormalization of the strong-coupling regime, only one bound state survives and $s$-wave scattering breaks scale invariance with a characteristic logarithmic dependence. The existence and order of magnitude of a critical coupling $\lambda_{(\mathrm{gs})}^{(*)}=1 / 4$ for $D=3$ 
are in agreement with recent experimental results $[10,11]$ for a wide range of polar molecules [26].

A final remark is in order. Strictly, even though a more careful treatment with dimensional regularization changes Eq. (17), the difference appears only at the level of the finite parts (linear in $\epsilon$ ) and is immaterial to the arguments presented here. These corrections, as well as a detailed treatment of the scattering sector of the theory, will be presented elsewhere.

This research was supported in part by CONICET and ANPCyT, Argentina (L. N.E., H. F., and C. A. G. C.) and by the University of San Francisco Faculty Development Fund (H.E.C.). The hospitality of the University of Houston and instructive discussions with Professor Carlos R. Ordóñez are gratefully acknowledged by H. E. C.

[1] K. M. Case, Phys. Rev. 80, 797 (1950).

[2] W. M. Frank, D. J. Land, and R. M. Spector, Rev. Mod. Phys. 43, 36 (1971), and references therein.

[3] G. Parisi and F. Zirilli, J. Math. Phys. 14, 243 (1973); C. Radin, J. Math. Phys. 16, 544 (1975); R. O. Mastalir, J. Math. Phys. 16, 743 (1975); 16, 749 (1975); 16, 752 (1975); H. van Haeringen, J. Math. Phys. 19, 2171 (1978).

[4] C. Schwartz, J. Math. Phys. 17, 863 (1976).

[5] S. P. Alliluev, Sov. Phys. JETP 34, 8 (1972).

[6] L. D. Landau and E. M. Lifshitz, Quantum Mechanics, Course of Theoretical Physics Vol. 3 (Pergamon Press, Oxford, 1977), pp. 114-117, 3rd ed.

[7] B. Simon, Arch. Ration. Mech. Anal. 52, 44 (1974); C. G. Simander, Math. Z. 138, 53 (1974); H. Narnhofer, Acta Phys. Austriaca 40, 306 (1974), and references therein.

[8] K. S. Gupta and S. G. Rajeev, Phys. Rev. D 48, 5940 (1993).

[9] C. G. Bollini and J. J. Giambiagi, Nuovo Cimento Soc. Ital. Fis. 12B, 20 (1972); G. 't Hooft and M. Veltman, Nucl. Phys. B44, 189 (1972); J.F. Ashmore, Nuovo Cimento Lett. 4, 289 (1972).

[10] J.-M. Lévy-Leblond, Phys. Rev. 153, 1 (1967); O. H. Crawford, Proc. Phys. Soc. London 91, 279 (1967), and references therein.

[11] C. Desfrançois, H. Abdoul-Carime, N. Khelifa, and J.P. Schermann, Phys. Rev. Lett. 73, 2436 (1994).

[12] E. Marinari and G. Parisi, Europhys. Lett. 15, 721 (1991).
[13] Momentum-space analyses of the two-dimensional $\delta$ function potential include C. Thorn, Phys. Rev. D 19, 639 (1979); K. Huang, Quarks, Leptons, and Gauge Fields (World Scientific, Singapore, 1982), Secs. 10.7 and 10.8; R. Jackiw, in M. A. B. Bég Memorial Volume, edited by A. Ali and P. Hoodbhoy (World Scientific, Singapore, 1991).

[14] A momentum-space renormalization-group analysis of the two-dimensional $\delta$-function potential is developed in S. K. Adhikari and T. Frederico, Phys. Rev. Lett. 74, 4572 (1995).

[15] Configuration-space analyses of the two-dimensional $\delta$ function potential include C. Manuel and R. Tarrach, Phys. Lett. B 328, 113 (1994); P. Gosdzinsky and R. Tarrach, Am. J. Phys. 59, 70 (1991); L. R. Mead and J. Godiness, Am. J. Phys. 59, 935 (1991).

[16] S. Coleman and E. Weinberg, Phys. Rev. D 7, 1888 (1973).

[17] See also S. K. Adhikari and A. Ghosh, J. Phys. A 30, 6553 (1997); S. K. Adhikari, T. Frederico, and I. D. Goldman, Phys. Rev. Lett. 74, 487 (1995).

[18] As an alternative, the momentum-space scheme of Ref. [14] is applied numerically to a related problem (inverse square potential with exponential screening) in C.F. de Araujo, Jr., L. Tomio, S. K. Adhikari, and T. Frederico, J. Phys. A 30, 4687 (1997).

[19] J. D. Louck and W. H. Shaffer, J. Mol. Spectrosc. 4, 285 (1960); J. D. Louck, J. Mol. Spectrosc. 4, 298 (1960); 4, 334 (1960).

[20] R. Jackiw, Phys. Today 25, No. 1, 23 (1972).

[21] Handbook of Mathematical Functions, edited by M. Abramowitz and I. A. Stegun (Dover Publications, New York, 1972).

[22] P. M. Morse and H. Feshbach, Methods of Theoretical Physics (McGraw-Hill, New York, 1953), Vol. 2, pp. $1665-1667$.

[23] I. S. Gradshteyn and I. M. Ryzhik, Table of Integrals, Series, and Products (Academic Press, New York, 1980).

[24] Incidentally, our theory in $D$ dimensions has a twodimensional appearance, due to dimensional transmutation. This is in sharp contrast with the behavior of the solutions of the wave equation and of the free-particle Schrödinger equation. See also Eq. (10).

[25] C. Quigg and J. L. Rosner, Phys. Rep. 56, 167 (1979).

[26] The present solution of the inverse square potential (with $l=0$ ) applies to the radial part of the wave function under a dipole potential; the angular part can be dealt with by using the method of Ref. [10]. 